\title{
L'enseignement des musiques du monde. Deuxième Symposium international, Bâle, octobre 1993
}

\section{Andreas Gutzwiller}

Traducteur : Isabelle Schulte-Tenckhoff

\section{OpenEdition \\ Journals}

Édition électronique

URL : http://journals.openedition.org/ethnomusicologie/1486

ISSN : 2235-7688

Éditeur

ADEM - Ateliers d'ethnomusicologie

Édition imprimée

Date de publication : 31 décembre 1994

Pagination : 311-313

ISBN : 2-8257-0503-9

ISSN : 1662-372X

Référence électronique

Andreas Gutzwiller, "L'enseignement des musiques du monde. Deuxième Symposium international, Bâle, octobre 1993 », Cahiers d'ethnomusicologie [En ligne], 7| 1994, mis en ligne le 03 janvier 2012, consulté le 04 mai 2019. URL : http://journals.openedition.org/ethnomusicologie/1486

Ce document a été généré automatiquement le 4 mai 2019.

Tous droits réservés 


\title{
L'enseignement des musiques du monde. Deuxième Symposium international, Bâle, octobre 1993
}

\author{
Andreas Gutzwiller \\ Traduction : Isabelle Schulte-Tenckhoff
}

1 Après la Vereniging voor Kunstzinnige Vorning (VKV) à laquelle revint l'organisation du premier symposium au Pays-Bas en juin 1992, c'est le Studio für aussereuropäische Musik de la Musik-Akademie de Bâle qui organisa la deuxième rencontre sur l'enseignement des musiques traditionnelles du monde.

2 La manifestation a attiré une centaine de participants venus du monde entier. Les ÉtatsUnis et le Royaume-Uni avaient envoyé chaqcun dix-neuf représentants, la Suisse quatorze, l'Allemagne dix, la France et la Hollande huit chacune, et, parmi les autres pays représentés, on trouvait la Suède (2), la Norvège (1), l'Inde (1) et la Malaisie (1).

3 Tous les participants étaient impliqués dans l'enseignement de tel ou tel secteur de l'éducation musicale, de l'école primaire au conservatoire. On a donc bénéficié d'une grande diversité d'approches, allant du pragmatisme des enseignants de musique aux démarches plus théoriques ou philosophiques des chercheurs.

4 L'allocution d'ouverture, sur le thème de l'évolution de la world music, fut prononcée par Robert E. Brown (Université de Californie à Los Angeles UCLA), qui souligna l'unité nécessaire de toutes les musiques. Elle fut suivie par un concert de diverses formations de gamelan balinais de la Musik-Akademie de Bâle.

5 Le lendemain matin commença le programme de travail proprement dit. La première séance, présidée par William P. Malm (Université de Michigan), fut consacrée aux problèmes et aux perspectives de l'incorporation des musiques du monde dans les programmes des universités et des conservatoires. La discussion tourna autour de deux questions : faut-il favoriser une introduction pratique ou plutôt théorique à de telles 
musiques, une introduction générale à un grand nombre de musiques ou plutôt un traitement approfondi de certaines d'entre elles?

La séance suivante, consacrée aux matériaux d'enseignement, fut présidée par Keith Howard (School of Oriental and African Studies), remplaçant Ki Mantle Hood (Université de Baltimore) absent pour des raisons de santé. L'exposé et la discussion ont clairement démontré que, si un certain progrès a été réalisé, beaucoup reste encore à faire.

Pendant toute l'après-midi, des ateliers et des démonstrations se déroulèrent sur des thèmes aussi variés que «La musique chinoise en salle de classe » (Jennifer Walden, École internationale de Kuala Lumpur) ou la « Musique de mbira » (Mark Grimshaw, University College Salford). En cours public, Huib Schippers (World Music School Amsterdam) donna un cours destiné aux élèves de sitar vivant à Bâle.

Peter Cooke (University of Edinburgh), T. Temple Tuttle (Cleveland State University) et Peter van Amstel (Koorenhuis, La Haye) présentèrent des programmes informatisés relatifs à l'apprentissage interactif, adaptable à la fois au système IBM et au système Macintosh. Trevor Wiggins (Dartington College of Arts) et Huib Schippers (VKV, Utrecht) exposèrent quelques idées pour l'élaboration d'une série intégrée de programmes interactifs permettant d'explorer la world music à divers niveaux de l'enseignement musical.

9 En soirée, on put apprécier de la musique d'Azerbaïdjan (Jeffrey Werbock) et du Baloutchistan (Jean During) puis, après un grand saut par-dessus l'Atlantique, des romances équatoriennes (Jorge Lopez Palacio) et du tango argentin (Sexteto Canyengue, Conservatoire de Rotterdam).

10 La première séance du samedi, présidée par Robert Brown (UCLA), fut consacrée à l'apprentissage pratique avancé, avec une introduction de Joep Bor du Conservatoire de Rotterdam, sur « World music - l'étape suivante ». La seconde séance, animée par Michael Koechlin (Radio Suisse DRS), fut consacrée aux projets à l'échelle municipale.

De nombreux ateliers prirent place à nouveau l'après-midi, centrés cette fois-ci sur des activités comprenant des enfants : gamelan balinais et musique indienne (Karl Richter et Ken Zuckerman, Musik-Akademie Basel), musique africaine avec Joseph Mataré (Bâle). Suivit un débat sur l'enseignement des musiques du monde dans les écoles primaires, animé par Huib Schippers. En cours public, Andreas Gutzwiller (Musik-Akademie Basel) fit une démonstration de son enseignement de shakuhachi.

12 Le concert du soir fut consacré à la musique indienne, avec Amelia Cuni (chant dhrupad), Philippe Brugière (rudra-vina), Steve Gorn (bansuri) et Arvind Parikh (sitar) - George Ruckert (MIT, Boston) assumant le rôle de maître de cérémonies.

13 Le dimanche matin, pour clore le symposium proprement dit, un débat fut organisé sur les méthodes d'enseignement, à savoir : méthodes non occidentales dans l'enseignement de la musique occidentale et vice-versa. Joan ten Hoonte (Conservatoire de Rotterdam) présenta un projet hollandais d'enseignement de la world music dans les écoles aux PaysBas, et Paul van den Bos (Conservatoire d'Alkmaar) donna une conférence encourageant la réflexion sur la manière dont les méthodes d'enseignement occidentales et les méthodes « world » peuvent bénéficier les unes des autres.

14 Le débat final de l'après-midi, animé par Keith Howard, permit de passer en revue et de résumer les trois thèmes principaux du symposium :

- cours d'introduction dans le secondaire et les conservatoires ; 
- l'enseignement des musiques du monde dans l'instruction publique

- la formation des musiciens et des enseignants. Dominique Vellard (Schola Cantorum Basiliensis) présentèrent "Deux univers de musique modale» en l'église St. Alban, jouant et chantant des raga indiens et de la musique médiévale.

17 Pendant la réunion du comité sous la présidence d'Arvind Parikh (Bombay), on débattit des diverses possibilités en vue d'établir un réseau d'échange d'informations et d'idées. Il fut décidé de renoncer à fonder une organisation de plus, mais de favoriser plutôt la simplicité en gardant le contact au moyen d'un bulletin trimestriel, que Huib Schippers proposa le publier à travers la VKV à Utrecht.

Au-delà de la diversité des exposés et des points de vue exprimés dans la discussion, tous les participants eurent le sentiment que l'enseignement des musiques du monde ne peut plus être considéré comme un phénomène marginal. La présence de musiques d'autres cultures à tous les niveaux d'enseignement en Occident, du primaire à l'université en passant par le conservatoire, est une réalité sociale et artistique des années quatre-vingtdix. L'échange de vues et d'idées réalisé à Bâle est un pas de plus vers la reconnaissance future et le perfectionnement de l'enseignement des musiques contemporaines et traditionnelles $\mathrm{du}$ monde entier dans les institutions occidentales consacrées à l'instruction musicale. 